\title{
BRECHA SALARIAL POR GÉNERO EN LOS MERCADOS DE TRABAJO PÚBLICOY PRIVADO EN MÉXICO
}

Reyna Rodríguez-Pérez Universidad Autónoma de Coahuila México

Raúl Ramos-Lobo Universidad Autónoma de Coahuila México

David Castro-Lugo Universidad Autónoma de Coahuila México 
Panorama Económico, Vol. 25 - No. 2 (Abril - Junio de 2017), pp. 149-172

\author{
Reyna Rodríguez-Pérez \\ Raúl Ramos-Lobo \\ David Castro-Lugo
}

\title{
Brecha salarial por género en los mercados de trabajo público y privado en México
}

\section{Resumen}

Esta investigación analiza la brecha salarial por género para el mercado de trabajo de México, teniendo en consideración diferentes segmentos: sector público y privado (formal e informal), antes y después de la crisis económica de 2009. Se utiliza el método de descomposición de Oaxaca-Blinder, incorporando una extensión de la metodología propuesta por Juhn-Murphy-Pierce para analizar los microdatos de la Encuesta Nacional de Ocupación y Empleo (ENOE) de 2005, 2009 y 2015. Los resultados indican que la brecha salarial de género se redujo, previa y posterior a la crisis, en todos los segmentos analizados. La fuente principal de esta evolución es la parte no explicada, acentuándose en el sector informal.

Palabras clave: Mercado laboral, Brechas Salariales, Empleo Formal, Empleo Informal. Nivel salarial y estructura.

Clasificación JEL: J3, J16, J31.

\section{Gender wage gap in the private and public labor markets in Mexico}

\begin{abstract}
This research analyze the wage gap by gender in the mexican labor market, taking into consideration the public and private (formal and informal) sectors, prior and after the 2009 economic crisis. An extension of the Oaxaca-Blinder decomposition method proposed by Juhn-Murphy-Pierce is used to analyze microdata of the Mexican Household Survey for the years 2005, 2009, and 2015. Results indicate that the wage gap by gender declined in all segments, prior and after the crisis.
\end{abstract}

Keywords: Labor markets, wage gaps, formal employment, informal jobs.

JEL Classification: J3, J16, J31.

\section{Écart salarial entre hommes et femmes sur les marchés du travail privés et publics au Mexique}

\section{Résumé}

Cette recherche analyse l'écart salarial entre hommes et femmes sur le marché du travail mexicain. Il tient en compte des secteurs public et privé (formels et informels), avant et après la crise économique de 2009. Une extension de la méthode de décomposition Oaxaca-Blinder proposée par JuhnMurphy-Pierce est utilisée pour analyser les microdonnées de l'enquête sur les ménages mexicains pour les années 2005, 2009 et 2015. Les résultats indiquent que l'écart de rémunération par sexe a diminué dans tous les segments. et après la crise.

Mots-clés: Marché du travail, écarts salariaux, emploi formel, emploi informel. Niveau de salaire et structure.

Nomenclature JEL: J3, J16, J31. 


\section{Brecha salarial por género en los mercados de trabajo público y privado en México}

INFORMACIÓN DEL ARTíCULO

Recepción de artículo: 07/02/2017

Concepto de evaluación: 20/002/2017

Aceptación de artículo: 28/03/2017
Reyna Rodríguez-Perez* Universidad Autónoma de Coahuila Mexico

Raúl Ramos-Lobo Universidad Autónoma de Coahuila Mexico

David Castro-Lugo Universidad Autónoma de Coahuila Mexico

\section{INTRODUCCIÓN}

Según el informe del Foro Económico Mundial $(2015)^{1}$, México se encuentra en una posición intermedia (71/145) en el ranking de los países analizados en relación a las diferencias de género. Si bien en algunas dimensiones como, por ejemplo, la salud o algunos indicadores relativos a la educación primaria y secundaria se encuentra en la primera posición del ranking, en otras dimensiones como la participación laboral y la brecha salarial se sitúa en las últimas posiciones: $121 / 145$ y 128/145, respectivamente.

Según el Censo de Población de 2010 (INEGI, 2010), el 51.2 \% de la población total en México son mujeres, de las cuales el $42.5 \%$ forman parte de la población económicamente activa, y de este universo el $94.8 \%$ se encuentran ocupadas. Por el rol de doble jornada que cumplen las mujeres, no es de extrañarse que la participación femenina se haya concentrado en actividades del sector terciario, de las cuales, el $31.7 \%$ son comerciantes, el $27.4 \%$ son trabajadoras en servicios personales y el $14.4 \%$ son oficinistas (ENOE, 2014), es decir, que participen en sectores considerados "flexibles" en términos de jornada, horario, pero también de menores ingresos. De hecho, su nivel de ingreso en promedio equivale al 80 \% respecto al hombre (Rodríguez y Castro (2014a)). La crisis de 2009 impactó desfavorablemente sobre la actividad económica del país. El producto interno bruto se contrajo más del 6\%, se perdieron más de 500 mil empleos, y los salarios tuvieron un fuerte retroceso en términos reales. Frente a la inexistencia de un sistema de seguro por desempleo, muchos trabajadores buscan su sobrevivencia en trabajos informales (sin

\footnotetext{
* Autor para correspondencia

Correos electrónicos: reynarodriguez@uadec.edu.mx*,rramos@ub.edu,david.castro@uadec.edu.mx

$1 \mathrm{http}$ //reports.weforum.org/global-gender-gap-report-2015/economies/\#economy=MEX (enlace valido el 8/6/2016)
} 
acceso a prestaciones laborales y seguridad social). Desafortunadamente, la condición de mujer es un elemento adicional de desventaja, ya que frecuentemente las organizaciones prefieren contratar hombres y, si la mujer desea acceder a un puestolaboral, podría verseen la necesidad de sacrificar aspiraciones salariales, aún y cuando esto no corresponda a una diferencia en la capacidad productiva entre hombres y mujeres que se refleje en diferencias en productividad (Rodríguez y Castro 2014a).

Los estudios sobre desigualdad salarial por género para México indican que hasta antes de 2009 la brecha salarial mostraba una tendencia a la reducción o en su caso no había crecido; Sin embargo, ante la crisis económica más fuerte de las últimas décadas, es válido preguntarnos, si las perturbaciones económicas impactan diferencialmente a los salarios por género. Estudios para otros países que analizan esta relación indican que las contracciones económicas genera una modificación en la brecha salarial por género, aunque no existe consenso sobre la dirección del efecto. O'Neill, 1985; King y Owens, 2009; Ghosh, 2013; Biddle y Hamermesh, 2013, y De la Rosa, 2014 encuentran evidencias a favor de una relación negativa entre recesión y brecha salarial (comportamiento contracíclico); Park y Shin, 2005; Kendil y Woods, 2009; King y Owens, 2009; Filio, 2010; Ghosh, 2013; Peña-Boquete, 2014; Nagore, 2015, y Razzu y Sangleton, 2016, apoyan la existencia de una relación positiva entre actividad económica y brecha salarial (comportamiento procíclico). Un segundo interrogante es si existen distintos comportamientos en el mercado de trabajo público y privado y dentro de este último, en empleos formales e informales.
Así pues, el objetivo de esta investigación es analizar las diferencias salariales por género para México en tres segmentos del mercado de trabajo con distintos mecanismos de determinación salarial: público, privado formal y privado informal, comparando los resultados obtenidos antes y después de la crisis de 2009.

La fuente de información que se emplea es la Encuesta Nacional de Ocupación y Empleo (ENOE) para los años 2005, 2009 y 2015 emitidas por el Instituto Nacional de Estadística y Geografía (INEGI). Se utiliza el método de descomposición Oaxaca-Blinder (1973), complementado con la metodología propuesta por Juhn-Murphy-Pierce (1991, 1993) que permite realizar la descomposición del diferencial y sus componentes explicativos a través del tiempo.

Los resultados obtenidos indican que la brecha salarial de género se redujo, previo y posterior a la crisis, en todos los segmentos del mercado laboral analizados, especialmente en el sector privado informal, lo que nos permite afirmar que a partir de los resultados obtenidos, no es posible sostener la presencia de una brecha salarial con comportamiento cíclico para el caso concreto de México. La fuente principal de la reducción es la parte no explicada, mientras que en la parte explicada los factores relevantes corresponden a las dotaciones de educación. Los resultados también indican la persistencia de diferencias salariales en contra de las mujeres no asociadas a dotaciones de factores, especialmente en el sector informal.

El resto del trabajo se estructura en cuatro apartados. El primero hace una breve presentación de los efectos de la crisis de 2009 en México, una discusión sobre la brecha salarial y recesión; así como la revisión de la evidencia empírica; en 
siguiente apartado aborda la metodología y datos empleados; el tercer apartado recoge nuestro análisis empírico sobre la desigualdad salarial por género y su descomposición en los mercados público, formal e informal. Por último, el trabajo concluye resumiendo las principales conclusiones obtenidas.

\section{REVISIÓN DE LITERATURA}

\subsection{La crisis económica de 2009 y su impacto en México}

Diversos estudios han señalado la sincronización de la economía mexicana al ciclo económico de Estados Unidos (EU), la cual se intensificó a partir de la firma del Tratado de Libre Comercio de América del Norte (TLCAN) en 1994. (Cuevas et al. 2003, Delajara 2012).

Los estudios sobre el proceso de integración económica entre México y EU, también indican que este proceso se ha presentado fundamentalmente en el sector manufacturero y dentro de este destacan la industria del papel y editorial, química, de minerales y de maquinaria, indicando que la integración se ha dado mediante la intensificación del intercambio de bienes comerciales. Cuevas et al (2003); Félix y Dávila (2008), y Delajara (2012).

Lo anterior implica que las recesiones económicas en Estados Unidos generarán un mayor impacto sobre la economía de México, tal como se observó en la crisis de 2008-2009 donde la actividad económica de México se contrajo más de 6.0 por ciento en 2009, y en el caso de las manufactura la reducción fue superior al 8 por ciento. Gráfica 1.

\subsection{La brecha salarial y la recesión.}

¿Las recesiones económicas tienen efectos diferenciales por género en el mercado laboral? ¿Debería alterarse la brecha salarial durante las recesiones?, si es así, ¿cuál debería ser el comportamiento por género? Desde una perspectiva teórica neoclásica se puede decir que no existen elementos para establecer a priori una

\section{Gráfica 1. México y Estados Unidos. Tasas de variación anual del Producto Interno Bruto (PIB) total y manufactura, 2005-2014. Porcentaje}

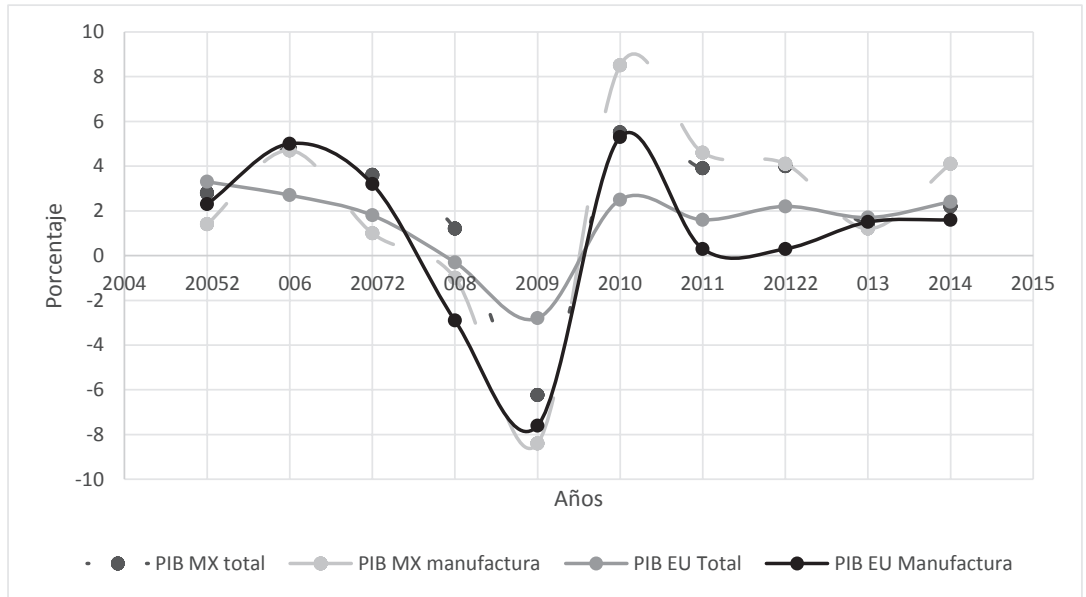

PIB: Producto Interno Bruto; MX: México; EU: Estados Unidos.

Fuente: México, Cuentas Nacionales, INEGI. Estados Unidos, Bureau of Economic Analysis. 
diferencia en el comportamiento de los salarios entre hombres y mujeres por la condición de sexo; sin embargo, estudios en diferentes periodos y países desde 1985 han encontrado evidencias que indican que las recesiones y el comportamiento del ciclo económico tiene efectos diferenciales por género, impactando sobre la brecha salarial. No obstante, es relevante señalar que para el caso de México son prácticamente inexistentes los documentos que analizan los efectos del ciclo económico sobre la brecha salarial.

Los argumentos para establecer un comportamiento heterogéneo del mercado laboral entre hombres y mujeres durante las recesiones son diversos, mismos que ayudan a explicar el aumento o reducción de la brecha salarial por género.

Dentro de estos argumentos podemos identificar básicamente 4: i) existencia de diferencia por género en la cobertura de los contratos de negociación colectiva (O'Neill, 1985, King y Owens, 2009; De la Rosa, 2014 y Ghosh, 2013); ii) distintas dotaciones de calificación y capital específico por género (Kendil y Woods, 2009); y iii) segmentación de los mercados de trabajo y distinta sensibilidad de los sectores al ciclo económico (Park y Shin, 2005; King y Owens, 2009; Filio, 2010; Ghosh, 2013; Peña-Boquete, 2014, Nagore, 2015, y Razzu y Sangleton, 2016), iv) modificaciones en la composición de la fuerza laboral derivados de la crisis y cambios en el comportamiento discriminatorio (Biddle y Hamermesh, 2013).

O'Neill (1985) es uno de los estudios pioneros que analiza el comportamiento de la brecha salarial durante las recesiones; analiza el periodo 1955-1982 y encuentra que un incremento de $1 \%$ en la tasa de desempleo genera un aumento significativo de
$0.008 \%$ en la brecha salarial hombres-mujer, lo que indicaría que el salario de las mujeres es más flexible que el de los hombres durante las recesiones; en otras palabras las mujeres tienen una curva de oferta más inelástica que la de los hombres durante las recesiones, incrementando la brecha salarial. El autor establece que la causa de dicho comportamiento se sustenta en el hecho de que el salario de las mujeres tiene menor cobertura de contratos de negociación colectivos que los hombres, percepción que es compartida por King y Owens, (2009); De la Rosa, (2014) y Ghosh, (2013), lo que llevaría a establecer una comportamiento contra-cíclico de la brecha salarial, es decir una relación inversa entre la actividad económica y brecha salarial.

Kandil y Woods (2002) por su parte, apoyados en el argumento de la diferencia en dotaciones, encuentran que los hombres disponen de mayor capital específico que las mujeres, lo que hace que prefieran mantener su empleo durante la recesión, aunque afecte su salario, lo que permitiría preservar su capital específico, implicando con ello la existencia de una curva de oferta laboral masculina relativamente inelástica, lo que llevaría a un comportamiento procíclico de la brecha salarial, es decir una relación directa entre el comportamiento de la actividad económica y la brecha salarial.

Apoyados en el argumento de segmentación de los mercados de trabajo y distinta sensibilidad de los sectores al ciclo económico, Park y Shin, (2005); Nagore, (2015); y Razzu y Sangleton, (2016), sostienen que los sectores u ocupaciones donde los hombres tienen una presencia predominante (manufactura, construcción, operarios, obreros, etc.), tienen un comportamiento más cíclico, lo que llevaría a incrementar el desempleo e impactar sobre las remu- 
neraciones durante la recesión; mientas que Peña-Boquete (2014); encuentra que las mujeres se ubican en sectores menos cíclicos, lo que implica una menor pérdida de empleos durante la recesión, en ambos casos, se espera un comportamiento pro-cíclico.

Por su parte King y Owens, (2009); Filio (2010), y Ghosh, (2013), afirman que las mujeres se ubican en actividades exportadoras, con mayor flexibilidad y peores condiciones laborales, hecho que las vuelve más sensibles a los entornos globales y son mayormente afectadas durante la contracción económica, llevando a observarse un comportamiento contra-cíclico.

Finalmente Biddle y Hamermesh, (2013), argumentan que el ciclo económico puede tener un claro efecto sobre la evolución de la brecha salarial. En primer lugar, mediante cambios en la composición de la fuerza laboral y en segundo lugar, asociado a cambios en el comportamiento discriminatorio de los empleadores, vinculado con el aumento del desempleo en periodos de crisis. La intuición detrás del modelo teórico de Biddle y Hamermesh (2013) es que un aumento en la ratio de desempleados por vacante ofrece un mayor margen a los empleadores para llevar a cabo acciones discriminatorias. La implicación de un comportamiento como el señalado anteriormente indicaría la presencia de un comportamiento contra cíclico, es decir la brecha salarial aumentaría cuando la actividad económica se encuentra en recesión.

Lo anterior permite establecer algunas precisiones: i) no obstante la ausencia de un marco teórico lo suficientemente desarrollo que permita explicar la presencia de diferencias salariales por género durante la recesión, sí existen argumentos que establecen un comportamiento de la brecha salarial durante el ciclo económico, espe- cialmente durante la recesión, ii) no existe consenso entre los argumentos en el sentido del comportamiento de la brecha salarial durante la recesión, mientras unos estudios encuentran evidencia empírica de una relación directa entre la brecha salarial y la actividad económica (pro-cíclico), otros aportan evidencias de una relación inversa (contra-cíclico), y iii) a pesar de que existe una amplia literatura sobre la brecha salarial por género, los estudio que analizan el comportamiento de la brecha salarial en el ciclo económico y particularmente en la recesión son relativamente escasos. Las precisiones anteriores permites establecer la necesidad de avanzar en la comprensión del comportamiento de la brecha salarial por género en los diferentes entornos económicos.

\subsection{Evidencia empírica para México.}

México constituye un caso de estudio relevante para analizar la brecha salarial, dado que se han argumentado diferentes motivos para analizar este aspecto del mercado laboral. En primer lugar, el proceso de apertura comercial iniciado a mediados de la década de los ochentas con la incorporación al GATT y el reforzamiento con el TLCAN ha sido uno de los motivos principales. Alarcón y McKinley (1994) y Brown et al (1999) son algunos de los estudios que analiza la brecha salarial dentro de este periodo. El periodo de apertura se estudia para conocer, sí a partir del ajuste estructural, la brecha salarial por sexo presenta cambios. A pesar de que utilizan diferentes fuentes de información y metodologías, ${ }^{2}$ los resultados no difieren: encuentran que la

\footnotetext{
2 Alarcón y McKinley (1994) utilizan la Encuesta Nacional de Ingreso Gasto de los Hogares (ENIGH) 1984, 1989 y 1992, y la metodología Oaxaca-Blinder, mientras que Artecona y Cunningham (2002) la Encuesta Nacional de Empleo Urbano (ENEU) y ENESTYC (1987-1993) y aplican la metodología de diferencias en diferencias.
} 
brecha salarial por género no disminuyó y que las mujeres trabajadoras fueron poco beneficiadas por el cambio estructural y la brecha salarial aumentó durante la apertura comercial. En cambio, Artecona y Cunnigham (2002) encuentran que en aquellos sectores más expuestos a la liberalización comercial, la brecha salarial disminuyó.

En segundolugar, la creciente participación de las mujeres en el mercado laboral y su inserción en el mismo, ha sido otro de los factores que han llevado a algunos autores a analizar el comportamiento de la brecha salarial. En concreto, Del Razo (2003); Brown y Domínguez (2007) y Calónico y Ñopo (2008) argumentan que la presencia de segregación ocupacional es uno de los principales elementos que explican la brecha salarial por encima del posible impacto de la apertura comercial. En este sentido, es importante destacar que, a diferencia de la evidencia obtenida para la mayoría de países desarrollados, Calónico y Ñopo (2008) encuentran evidencia de que la segregación horizontal favorece a las mujeres, sin embargo, la segregación vertical y jerárquica penaliza a las mujeres y ambos fenómenos están claramente interrelacionados.

Otro factor apuntado recientemente para analizar el comportamiento de la brecha salarial es la creciente presencia de informalidad laboral en el país. Popli (2013) analiza el periodo 1996-2006 y separa entre trabajadores formales e informales, identificando distintas dinámicas en la distribución salarial de uno y otro grupo de trabajadores.

El reconocimiento de la existencia de potenciales efectos regionales y sectoriales generados por la apertura comercial llevaron a considerar estos elementos en el análisis de la brecha salarial realizado por Rodríguez y Castro (2014b) y Castro et al (2015).

Teniendo en cuenta estos antecedentes, el presente estudio tiene como objetivo analizar si la brecha salarial presenta modificaciones relevantes en periodos de crisis y si este comportamiento es distinto por tipos de mercados (público y privado distinguiendo entre sector formal e informal). Para ello, se determinan las diferencias salariales por género $y$ se realiza la descomposición en atributos y la parte no explicada por los mismos, así como los cambios en la desigualdad y su comportamiento a través del tiempo.

\section{METODOLOGÍA Y DATOS}

\section{1.- Metodología}

En el análisis empírico se utilizan dos metodologías de descomposición de los salarios que permiten identificar qué parte de la brecha salarial se debe a diferencias en las características observables entre hombres y mujeres y la parte vinculada con diferencias en los rendimientos asociados a dichas características.

En primer lugar, se aplica la metodología propuesta por Oaxaca (1973) y Blinder (1973) y, en segundo lugar, la metodología de Juhn-Murphy-Pierce (Juhn et al, 1993). Para poder aplicar ambos métodos es necesario identificar los factores determinantes de los salarios individuales para cada año y segmento analizado a partir de una ecuación de Mincer como la siguiente:

$$
w_{i}=X_{i} \beta+\varepsilon_{i}
$$

Donde $\mathrm{w}_{\mathrm{i}}$ representa el salario por hora del trabajador i en logaritmos y $\mathrm{X}_{\mathrm{i}}$ denota 
distintas características individuales y del puesto de trabajo. En concreto, se consideran los años de estudio del individuo, su experiencia potencial en el mercado de trabajo ${ }^{3}$ y su cuadrado, el tipo de jornada (tiempo completo o tiempo parcial), el tipo de contrato, la ocupación y la región de residencia. Por último, $\beta$ es un vector de parámetros y $\varepsilon_{\mathrm{i}}$ es un término de error aleatorio.

La ecuación (1) se estima por mínimos cuadrados ordinarios robustos a la presencia de heteroscedasticidad tanto para la muestra conjunta de hombres y mujeres como para cada uno de los dos segmentos por separado. A partir de dichas estimaciones es posible descomponer la diferencia en el salario promedio entre hombres y mujeres $(\Delta)$ tal y como se muestra a continuación:

$$
\begin{aligned}
\Delta= & \left(W^{h}-W^{m}\right)=\left(X^{h}-X^{m}\right) \beta^{*}+ \\
& \left\{X^{h}\left(\beta^{m}-\beta^{*}\right)+X^{h}\left(\hat{\beta}^{*}-\beta^{m}\right)\right\}
\end{aligned}
$$

Donde $W^{h}$ y $W^{m}$ son los salarios medios de hombre y mujeres; $X^{h}$ y $X^{h}$ son las características observadas medias para cada colectivo y $\beta^{h}, \beta^{m}$ y $\beta^{*}$ son los coeficientes estimados para hombre y mujeres y para el pool de ambos colectivos, respectivamente.

El primer componente del lado derecho de la ecuación (2) representa el efecto sobre el diferencial salarial promedio que se debe a las diferencias en las características (o componente "explicado"), mientras que el segundo corresponde al efecto de los coeficientes (o componente "no explicado"). Ambos componentes pueden descomponerse, a su vez, distinguiendo la aportación

3 Calculada como la edad del individuo menos los años de estudio completados -6 . asociada a cada variable explicativa individual al diferencial. Un problema habitual al aplicar este procedimiento es que la elección de una determinada categoría específica para las variables ficticias que forman parte del análisis (por ejemplo, ocupación) puede afectar a los resultados de la descomposición detallada tal y como demuestran Oaxaca y Ransom (1999). Por ese motivo, hemos seguido la estrategia de normalización de variables ficticias sugerida por Yun (2005), lo que permite superar el problema de identificación explicado y llevar a cabo una estimación adecuada de la contribución real de cada variable al diferencial.

Tal y como se argumenta en Simón et al. (2008), en este tipo de análisis se utiliza de manera habitual la estructura salarial de los hombres como referencia en la descomposición lo que implica suponer que en ausencia de discriminación esta estructura salarial sería también la correspondiente a las mujeres. Sin embargo, en nuestro análisis se ha optado por utilizar como referencia la estructura salarial conjunta de hombres y mujeres lo que tiene ciertas ventajas ya que es posible utilizar toda la información disponible para estimar los rendimientos salariales y además supone utilizar una aproximación más natural a la estructura salarial no discriminatoria de la economía analizada.

En lo que se refiere a la aplicación de la metodología de Juhn-Murphy-Pierce (Juhn et al, 1993), la principal diferencia respecto a la metodología anterior es que ahora el objetivo es explicar los cambios en el diferencial salarial entre hombres y mujeres en dos momentos distintos del tiempo. Así pues, y tomando como punto de partido el año 2005, el salario medio 
de los hombres $(m)$ en ese momento del tiempo se puede expresar como:

$$
w_{05}^{m}=X_{05}^{m} \hat{\beta}_{05}+\sigma_{05} \theta_{05}^{m}
$$

Donde $w_{05}^{s}$ es el salario medio por hora de los hombres expresado en logaritmos; $X_{05}^{m}$ representa los valores medios de las característicasindividuales delos hombres; $\hat{\beta}_{05}^{m}$ es el vector de parámetros estimados conjuntamente para hombres y mujeres a partir de la ecuación (1) en 2005; $\theta_{05}^{m}$ es el residuo salarial estandarizado promedio de los hombres; $\sigma_{05}$ es la desviación estándar de los residuos salariales de la muestra conjunta de hombres y mujeres. De manera similar, se podría representar la ecuación (3) de manera equivalente para las mujeres $(f)$.

Utilizando la estructura salarial estimada conjuntamente para hombres y mujeres como estructura salarial de referencia en la descomposición tal y como se ha explicado anteriormente, la diferencia salarial media entre hombres y mujeres en $2005\left(D_{05}\right)$ se expresa de acuerdo con la siguiente ecuación:

$$
\begin{aligned}
& D_{05}=w_{05}^{m}-w_{05}^{f}=\left(X_{05}^{m}-X_{05}^{f}\right) \hat{\beta}_{05}+ \\
& \left(\theta_{05}^{m}-\theta_{05}^{f}\right) \sigma_{05}=\Delta X_{05} \hat{\beta}_{05}+\Delta \theta_{05} \sigma_{05}
\end{aligned}
$$

Donde el operador $\Delta$ indica la diferencia entre hombres y mujeres en el promedio de la variable. La ecuación (4) permite identificar, de manera similar a la descomposición de Oaxaca-Blinder, la contribución a la diferencia salarial entre hombres y mujeres en 2005 de las diferencias de la parte explicada y de la parte no explicada. La primera parte de la expresión captura precisamente la parte explicada, mientras que el resto de términos está relacionado con la parte inexplicada. Así pues, el segundo término está relacionado con factores como habilidad inobservada y otras características inobservables que se aproximan a través de la diferencia en los residuos salariales estandarizados promedio de hombres y mujeres $\left(\theta_{05}^{m}-\theta_{05}^{f}\right)$ multiplicada por la dispersión de la distribución de los residuos $\left(\sigma_{05}\right)$ que determina la penalización salarial que sufre el colectivo que ocupa la posición más baja en la distribución de residuos.

La ecuación (4) puede adaptarse de manera similar para cualquier año en qué estemos interesados, por lo que si replicamos el análisis para el año más reciente para el que se realiza el análisis (2015) y restamos ambas ecuaciones, la variación en el diferencial salarial por género entre 2005 y 2015 se expresa como:

$$
\begin{gathered}
D_{15}-D_{05}=\left(\Delta X_{15}-\Delta X_{05}\right) \beta_{15}+\Delta X_{05}\left(\beta_{15}\right. \\
\left.-\beta_{05}\right)+\left(\Delta \theta_{15}-\Delta \theta_{05}\right) \sigma_{15}+\Delta \theta_{05}\left(\sigma_{15}-\sigma_{05}\right)
\end{gathered}
$$

De manera que, según la ecuación (5), la variación en la magnitud del diferencial salarial por género entre ambos 2005 y 2015 puede explicarse por cuatro factores: En primer lugar, que se modifiquen las características productivas de hombres y mujeres; en segundo lugar, que la estructura de diferenciales salariales de la economía experimente modificaciones. El tercer factor estaría relacionado con posibles cambios los factores inobservables que aproximen o alejen los residuos salariales promedio de hombres y mujeres. Por último, en cuarto lugar, es posible que haya cambios en la dispersión de los residuos salariales y, por lo tanto, en la penalización asociada a la diferencia en los residuos estandarizados promedio. Es interesante destacar que estos factores pueden agruparse entre aquellos que tienen un carácter específico de género 
(el primer y tercer componente) y los que recogen efectos asociados a características generales de la estructura salarial (el segundo y el cuarto componente). Por tanto, la suma del primer y segundo componentes corresponde al efecto conjunto de los factores asociados a las características productivas en los cambios experimentados por el diferencial salarial por género, mientras que la suma del tercer y cuarto componente al efecto conjunto de los factores inobservables.

\subsection{Datos}

El estudio se basa en el análisis de los datos individuales de distintas olas de la Encuesta Nacional de Ocupación y Empleo (ENOE). En concreto, se considera el tercer trimestre de 2005, 2009 y 2015. Con la selección de estos años se pretende cubrir el periodo de información disponible más reciente de la ENOE. El 2005 es un año estable y primera observación de la ENOE; la inclusión de 2009 permitirá captar el comportamiento de la brecha salarial por género durante la crisis económica de ese año y 2015 la situación una vez recuperada una coyuntura económica más favorable. La comparación de los niveles previos a la crisis, durante la crisis y posterior, permitirá identificar, en primera instancia, si se presenta un efecto diferenciado por sexo que genera un variación en la brecha salarial, y en segundo instancia, en caso de que se confirme la presencia de efectos heterogéneos por sexo, determinar si la relación es directa o inversa entre actividad económica y brecha salarial, así como identificar cuál de los argumentos explicativos presentados previamente son más relevantes para México para cada uno de los segmentos que se analizan.

La población de estudio son mujeres y hombres de 16 a 65 años de edad ocu- pados en el mercado laboral de México, identificando diferentes segmentos como el público y privado (formal e informal). En el mercado de trabajo privado formal se clasifican a todos aquellos que cuentan con seguridad social (salud y pensión); mientras que informales se consideran los que no cotizan y no cuentan con estas prestaciones laborales. En el sector público se incluye a todos aquellos que trabajan en administración pública federal, defensa y marina, sector paraestatal, escuelas, hospitales y servicios asistenciales, educativos y otras instituciones ${ }^{4}$.

Los salarios ${ }^{5}$ de los trabajadores son captados en forma mensual y convertidos a salario por hora y se considera trabajo a tiempo completo cuando la jornada laboral es igual o mayor a 40 horas a la semana.

Todos los cálculos realizados han considerado el factor de expansión facilitado por la propia encuesta.

\section{RESULTADOS}

\subsection{Análisis descriptivo}

El cuadro 1 presenta las principales características de los datos del tercer trimestre del 2005, 2009 y 2015, considerando la información agregada, así como por sectores (público, privado formal e informal). El salario promedio por hora favorece a los hombres, con excepción del sector público, aunque en todos los casos se aprecia una tendencia a la convergencia, por lo que la brecha por género ha seguido una tendencia decreciente a lo largo del periodo, sin que se aprecien cambios

4 No se consideran los trabajadores informales del sector público (alrededor del $5 \%$ de la muestra).

5 De los datos de la ENOE no es posible saber si los salarios reportados son brutos o netos, dado que no se especifica en la pregunta, por lo tanto se puede suponer que la gente reporta el ingreso disponible. 
significativos asociados a la crisis de 2009; en lo relativo a la jornada laboral por semana las mujeres trabajan menos horas, y no se observan una reducción significativa en el tiempo, aunque sí se aprecian diferencias importantes por sectores, siendo mayor en el privado informal y menor en el privado formal. En lo que se refiere al resto de variables, la diferencia en años educativos es a favor de las mujeres, especialmente en el sector público mientras que la experiencia favorece a los hombres, excepto 2015 en el sector privado informal. En promedio el 60 por ciento de mujeres y el 80 por ciento de hombres tienen jornadas laborales de tiempo completo, mientras que por sectores, el privado formal e informal presenta la mayor y menor proporción respectivamente. El 23 por ciento de ellas son jefas de familia, aunque la tendencia es creciente a lo largo del periodo de estudio. Un resultado a destacar es que existe una mayor proporción de ocupados de ambos sexos en el sector informal, aunque también en este sector presenta la menor escolaridad promedio. Las mujeres tienen una mayor participación en actividades públicas respecto a los hombres, aunque las diferencias más grandes en participación por sexo se encuentran en el mercado de trabajo privado principalmente en el formal. Finalmente es relevante destacar que entre los diferentes sectores, público y privado (formal e informal) se aprecian diferencias importantes en las diferentes variables observadas, aportando elementos para su análisis por separado. (Cuadro 1).

\subsection{Desigualdad salarial por hora y su descomposición para mujeres y hombres}

Una vez hecha una breve revisión de las características del mercado laboral, se procede a la realizar la descomposición de la diferencia salarial, e identificar la parte explicada y no explicada por los atributos de capital humano.

Las tres primeras filas del cuadro 2 muestran la evolución de los salarios promedios por género así como de la brecha salarial, que tal y como se ha comentado anteriormente, muestra un comportamiento claramente decreciente en el período analizado, el cual se relaciona con una caída más que proporcional del salario promedio de los hombres. A continuación se presenta la información sobre las diferencias en la brecha salarial por sexo que corresponderían a las características de cada grupo, si los atributos fueran remunerados por igual a hombres y mujeres, es decir la parte explicada de la diferencia a partir de la descomposición de Oaxaca-Blinder. Estos resultados indican que en todo el periodo las mujeres deberían de percibir remuneraciones promedio superiores a las de los hombres en aproximadamente un 11.0 por ciento.

La mayor parte de la brecha no se explica por las características, sino por el efecto precio, aunque la participación de este componente muestra una reducción durante el periodo analizado. Dentro de los elementos que pueden estar capturados en la parte "no explicada" se encuentran las diferencias promedio en las capacidades innatas de las personas de ambos sexos, la diferencia en la calidad de la educación y la discriminación entre otros (véase Bertrand, 2011 o Eswaran, 2014, entre otros). Si consideramos que no existe evidencia para pensar que, en promedio, un determinado grupo tiene ventaja significativa sobre otro en aspectos como habilidades o dotaciones innatas, calidad de la educación, entre otros, este resultado nos permite afirmar que al 


\section{Cuadro 1.México. Características promedio por sexo, general y sectores (público, privado formal e informal), 2005, 2009 y 2015.}

\begin{tabular}{|c|c|c|c|c|c|c|}
\hline \multicolumn{7}{|c|}{ México } \\
\hline & \multicolumn{2}{|c|}{2005} & \multicolumn{2}{|c|}{2009} & \multicolumn{2}{|c|}{2015} \\
\hline VARIABLES & Mujer & Hombre & Mujer & Hombre & Mujer & Hombre \\
\hline Salario por hora & 35.52 & 38.24 & 34.86 & 36.02 & 32.51 & 32.50 \\
\hline horas & 38.59 & 45.87 & 37.88 & 45.15 & 37.84 & 45.44 \\
\hline Años educativos & 9.20 & 8.80 & 9.56 & 9.08 & 10.05 & 9.55 \\
\hline Experiencia & 20.37 & 21.32 & 21.17 & 21.59 & 21.98 & 21.61 \\
\hline Casado & 0.48 & 0.70 & 0.51 & 0.69 & 0.52 & 0.69 \\
\hline Jefe de hogar & 0.20 & 0.66 & 0.23 & 0.64 & 0.24 & 0.63 \\
\hline Tiempo completo & 0.61 & 0.80 & 0.59 & 0.78 & 0.60 & 0.79 \\
\hline Sector publico & 0.13 & 0.09 & 0.13 & 0.09 & 0.11 & 0.07 \\
\hline Sector privado formal & 0.26 & 0.28 & 0.25 & 0.27 & 0.27 & 0.30 \\
\hline Sector privado informa & 0.61 & 0.63 & 0.62 & 0.64 & 0.61 & 0.63 \\
\hline Observaciones & $11,104,788$ & $19,459,980$ & $12,703,162$ & $20,667,183$ & $13,908,143$ & $22,494,136$ \\
\hline \multicolumn{7}{|c|}{ Sector Publico } \\
\hline Salario por hora & 67.14 & 62.45 & 63.74 & 61.36 & 59.41 & 58.45 \\
\hline Horas & 36.42 & 42.67 & 36.6 & 42.2 & 37.11 & 41.96 \\
\hline Años educativos & 13.5 & 12.52 & 13.99 & 12.79 & 14.41 & 13.64 \\
\hline Experiencia & 19.53 & 22 & 20.02 & 22.11 & 20.21 & 22.05 \\
\hline Casado & 0.57 & 0.82 & 0.55 & 0.79 & 0.57 & 0.79 \\
\hline Jefe de hogar & 0.21 & 0.81 & 0.27 & 0.77 & 0.28 & 0.76 \\
\hline Tiempo completo & 0.53 & 0.73 & 0.58 & 0.73 & 0.58 & 0.74 \\
\hline Observaciones & $1,435,898$ & $1,757,711$ & $1,668,795$ & $1,895,011$ & $1,573,551$ & $1,652,208$ \\
\hline \multicolumn{7}{|c|}{ Sector privado formal } \\
\hline Salario por hora & 34.85 & 39.54 & 33.28 & 37.3 & 31.06 & 33.66 \\
\hline Horas & 45.16 & 48.74 & 45.75 & 49.15 & 46.3 & 49.49 \\
\hline Años educativos & 10.57 & 10.11 & 11.1 & 10.39 & 11.44 & 10.82 \\
\hline Experiencia & 15.55 & 18.16 & 16.42 & 18.61 & 17.41 & 18.8 \\
\hline Casado & 0.39 & 0.70 & 0.42 & 0.70 & 0.45 & 0.70 \\
\hline Jefe de hogar & 0.17 & 0.65 & 0.20 & 0.64 & 0.22 & 0.62 \\
\hline Tiempo completo & 0.89 & 0.95 & 0.90 & 0.95 & 0.91 & 0.96 \\
\hline Observaciones & $2,916,855$ & $5,370,586$ & $3,159,923$ & $5,475,191$ & $3,791,783$ & $6,770,608$ \\
\hline \multicolumn{7}{|c|}{ Sector privado informal } \\
\hline Salario por hora & 29.09 & 34.22 & 29.38 & 31.88 & 28.2 & 28.89 \\
\hline Horas & 36.22 & 45.07 & 34.99 & 43.93 & 34.21 & 43.89 \\
\hline Años educativos & 7.695 & 7.694 & 7.996 & 8.015 & 8.635 & 8.464 \\
\hline Experiencia & 22.63 & 22.61 & 23.31 & 22.74 & 24.33 & 22.91 \\
\hline Casado & 0.50 & 0.68 & 0.54 & 0.68 & 0.54 & 0.67 \\
\hline Jefe de hogar & 0.21 & 0.64 & 0.24 & 0.63 & 0.25 & 0.61 \\
\hline Tiempo completo & 0.51 & 0.75 & 0.47 & 0.71 & 0.46 & 0.71 \\
\hline Observaciones & $6,752,035$ & $12,331,683$ & $7,874,444$ & $13,296,981$ & $8,542,809$ & $14,071,320$ \\
\hline
\end{tabular}

Fuente: Elaboración propia en base a los datos de la ENOE tercer trimestre 2005, 2009 y 2015. 
menos la mayor parte de las diferencias "no explicadas" parecen corresponderse a comportamientos discriminatorios.

El cuadro 2 también muestra las diferencias salariales en el mercado laboral del sector público. Los resultados indican que la brecha se encuentra a favor de las mujeres en todo el periodo analizado, a pesar de la caída del salario promedio de este grupo laboral. Los resultados de la descomposición de Oaxaca-Blinder muestran que los atributos explican la mayor proporción de las desigualdades. La parte no explicada atribuible a discriminación es relativamente baja, favoreciendo a los hombres en todos los periodos; lo que parece indicar que, a pesar de que las mujeres en promedio tienen percepciones superiores a los hombres, en la última década la discriminación salarial sobre las mujeres en el sector público en México ha aumentado.

En lo que se refiere a las diferencias salariales en el mercado del sector privado-formal, los resultados indican que en todo el periodo la brecha se encuentra en contra de las mujeres, con una leve tendencia decreciente, así al final del periodo de análisis la diferencia salarial promedio por género es de 9.0 por ciento. Este com-

Cuadro 2. México. Desigualdad salarial y su descomposición explicada y no explicada por sectores (público, privado formal e informal), 2005, 2009 y 2015.

(Los salarios por hora mensual están expresados a logaritmos)

\begin{tabular}{|l|c|c|c|}
\hline \multicolumn{1}{|c|}{ Total de la muestra } & 2005 & 2009 & 2015 \\
\hline Logaritmo del salario por hora femenino & 3.190 & 3.209 & 3.166 \\
\hline Logaritmo del salario por hora masculino & 3.281 & 3.254 & 3.195 \\
\hline Diferencia: Mujeres-Hombres & -0.0905 & -0.0448 & -0.0289 \\
\hline Parte Explicada & 0.101 & 0.111 & 0.108 \\
\hline Parte No Explicada & -0.191 & -0.156 & -0.137 \\
\hline Sector público & & & \\
\hline Logaritmo del salario por hora femenino & 3.985 & 3.974 & 3.890 \\
\hline Logaritmo del salario por hora masculino & 3.880 & 3.874 & 3.857 \\
\hline Diferencia: Mujeres-Hombres & 0.105 & 0.0998 & 0.0335 \\
\hline Parte Explicada & 0.150 & 0.148 & 0.108 \\
\hline Parte No Explicada & -0.0446 & -0.0485 & -0.0746 \\
\hline Sector privado - formal & & & \\
\hline Logaritmo del salario por hora femenino & 3.335 & 3.312 & 3.238 \\
\hline Logaritmo del salario por hora masculino & 3.449 & 3.413 & 3.331 \\
\hline Diferencia: Mujeres-Hombres & -0.114 & -0.101 & -0.0930 \\
\hline Parte Explicada & 0.0173 & 0.0338 & 0.0377 \\
\hline Parte No Explicada & -0.131 & -0.135 & -0.131 \\
\hline Sector privado -informal & & & \\
\hline Logaritmo del salario por hora femenino & 2.959 & 3.005 & 3.002 \\
\hline Logaritmo del salario por hora masculino & 3.122 & 3.100 & 3.053 \\
\hline Diferencia: Mujeres-Hombres & -0.163 & -0.0945 & -0.0511 \\
\hline Parte Explicada & 0.104 & 0.114 & 0.129 \\
\hline Parte No Explicada & -0.268 & -0.208 & -0.180 \\
\hline
\end{tabular}

Fuente: Elaboración propia en base a los datos de la ENOE tercer trimestre 2005, 2009 y 2015. 
portamiento se presenta en un entorno de contracción de los salarios reales promedio, donde los hombres resultan más afectados que las mujeres. Los resultados de la descomposición permiten apreciar que, de acuerdo a las dotaciones de factores, las mujeres deberían recibir un ingreso promedio superior al de los hombres, el cual se incrementa a lo largo de los años de estudio, indicando el crecimiento relativo de las dotaciones de factores de las mujeres en relación a los hombres. La parte no explicada muestra una condición desfavorable para las mujeres, que es superior al 10 por ciento. La magnitud del efecto precio determina el comportamiento de la brecha salarial total, en comparación con el sector público donde las diferencias en dotaciones explica la mayor parte de la brecha salarial.

Por último, y tal y como se puede observar en la parte inferior del cuadro 2, las diferencias salariales observadas en el mercado laboral privado informal se encuentran en contra de las mujeres, aunque la brecha de salarios disminuyó significativamente. Observando la fuente de la reducción, ésta se explica tanto por la reducción en los salarios de los hombres como por un aumento del salario promedio de las mujeres.

La descomposición de la brecha salarial permita apreciar que es el resultado de dos efectos. Por un lado, la parte explicada indica que en el sector informal las diferencias a favor de las mujeres en dotaciones de capital, como son educación y experiencia, se amplían, mientras que la magnitud de la parte no explicada, y que se asocia a discriminación, se reduce significativamente, no obstante es en el segmento del trabajo informal donde es mayor la brecha salarial por género asociada a la discriminación, hecho que resulta desafortunado, porque es en este grupo de trabajadores donde las remuneraciones son menores y hay una mayor ausencia de prestaciones sociales y laborales.

A manera de resumen de esta sección se pueden destacar algunos elementos: i) existen diferencias salariales importantes entre hombres y mujeres para todos los segmentos laborales analizados; ii) durante la última década el salario real promedio se ha reducido, afectando en mayor magnitud a los hombres en los distintos mercados laborales; iii) la brecha salarial muestra comportamientos distintos entre el sector público y privado; mientras que en el primero favorece a las mujeres, y la fuente de disparidad se sustenta en las dotaciones de factores, en el sector privado la brecha es a favor de los hombres, y la fuerte principal de la disparidad es atribuible al precio; iv) durante el periodo de análisis la brecha salarial se reduce significativamente, especialmente en el sector privado, siendo la reducción en la diferencias de remuneración a los factores la causa principal, hecho que posiblemente este asociado a una mayor competencia laboral y por tanto a una mayor penalización a las prácticas discriminatorias; v) finalmente, la brecha salarial presenta una tendencia decreciente, que no parece alterarse por el efecto de la crisis económica de 2009, lo que indicaría que para el caso de México durante la última década no se encuentra evidencias que apoyen la hipótesis propuesta de Park y Shing (2005) sobre el comportamiento procíclico de la brecha salarial.

\subsection{Descomposición del diferencial de salarios 2005-2009-2015}

En este apartado se muestran los resultados de la aplicación de la metodología Juhn-Murphy-Pierce $(1991,1993)$ sobre la 
descomposición del diferencial de salarios medios por género, identificando los cambios en la brecha salarial durante el periodo de estudio y la fuente de variación. En primer lugar se presentan los resultados para toda la muestra analizada y, a continuación, los diferentes segmentos del mercado de trabajo considerados en el estudio: público y privado (formal e informal).

El cuadro 3 presenta la información de la descomposición para toda la muestra, en los primeros tres renglones se muestra el diferencial salarial en el año correspondiente, así como la descomposición, es decir la parte explicada por las características y los residuos salariales que corresponden a la parte no explicada (donde un valor positivo indica que se trata de un indicador favorable para las mujeres). El renglón 4 presentan las variaciones en la brecha salarial en los subperiodos 2005-2009 y 2009-2015, donde se puede observar que en ambos lapsos, la brecha salarial se reduce, pero en mayor medida entre 2005 y 2009 , lo que parece mostrar que las perturbaciones económicas de 2009 implicaron modificaciones relevantes en el comportamiento de la brecha salarial, aunque en el periodo 2009-2015, no se presenta un cambio de tendencia, como se esperaría de acuerdo a un comportamiento procíclico.

La desagregación de las variaciones temporales entre la parte explicada y no explicada, renglones 5-6, permite establecer que es éste último componente el más relevante para explicar el comportamiento de la brecha salarial en el tiempo.

Los renglones 7-9 presentan la descomposición de la variación de la brecha salarial correspondiente al componente explicativo, renglón 5, el cual tiene un comportamiento desigual a lo largo del periodo analizado, contribuyendo a reducir la brecha en el lapso 2005-2009, y aumenta en el segundo subperiodo.

El comportamiento de los renglones 7-9 permite identificar que a lo largo de todo el periodo los factores asociados a las características actuaron en favor de la reducción de la brecha salarial, mientras que los precios solo actuaron en esa dirección en el primer periodo.

Los renglones 10-16 del cuadro 3 muestran el aporte individual de cada característica a la variación de la brecha salarial, y donde se puede destacar que en el caso de la educación, la mayor dotación de las mujeres favoreció la reducción de la brecha salarial, pero el precio de este factor actuó en dirección contraria, mismo comportamiento observado en el caso del factor jefe de hogar.

La consideración por subperiodos permite apreciar modificaciones relevantes en el comportamiento de algunos factores, con una tendencia hacia el aumento de la brecha salarial durante el lapso 2009-2015.

El cuadro 4 presenta los resultados de la descomposición relativos al sector público, donde se observa una brecha salarial a favor de las mujeres, la cual se mantiene sin variaciones relevantes durante el primer subperiodo, pero en el segundo (2009-2015) se presenta una reducción significativa, fundamentalmente asociado a la características, que presentan una modificación favorable a los hombres tanto en precio como en dotaciones, especialmente en los factores educativos y horas trabajadas, tal como se aprecia en los renglones 10-12

En lo que se refiere a la descomposición y los cambios del diferencial de salarios 


\section{Cuadro 3. México. Descomposición Juhn-Murphy-Pierce del diferencial salarial por razón de género. 2005-2009-2015. \\ (Los salarios por hora mensual expresados a logaritmos)}

\begin{tabular}{|c|c|c|c|c|c|c|c|c|}
\hline & & \multicolumn{2}{|c|}{2005} & 2009 & 2015 & & & \\
\hline \multicolumn{3}{|c|}{ Diferencial salarial por sexo (1) } & -.0904513 & -.0448498 & -.028919 & & & \\
\hline \multicolumn{3}{|c|}{ Características observadas (2) } & .1345232 & .1400186 & .1243426 & & & \\
\hline \multicolumn{3}{|l|}{ Residuos salariales (3) } & -.2249745 & -.1848684 & -.1532616 & & & \\
\hline & & & & 2005-2009 & 2009-2015 & & & \\
\hline \multicolumn{3}{|l|}{ Diferencias (4) } & & .0456015 & .0159308 & & & \\
\hline \multicolumn{3}{|l|}{ Explicada (5) } & & .0054954 & -.015676 & & & \\
\hline \multicolumn{3}{|l|}{ No Explicada (6) } & & .0401061 & .0316068 & & & \\
\hline \multicolumn{3}{|l|}{ Características (Q) (7) } & & .0113656 & .0158856 & & & \\
\hline \multicolumn{3}{|l|}{ Precios (P) (8) } & & -.0056646 & -.0312496 & & & \\
\hline \multicolumn{2}{|l|}{ QP (9) } & & & -.0002057 & -.000312 & & & \\
\hline & \multicolumn{4}{|c|}{ 2005-2009 } & \multicolumn{4}{|c|}{ 2009-2015 } \\
\hline & $E$ & Q & $\mathrm{P}$ & QP & E & Q & $\mathrm{P}$ & QP \\
\hline Total (10) & .0054954 & .0113656 & -.0056646 & \begin{tabular}{l|l}
6 & -.0002057
\end{tabular} & -.015676 & .0158856 & -.031249 & -.000312 \\
\hline Horas (11) & 0.004240 & -.0000281 & .0042689 & $-8.05 e-07$ & .0055148 & .0069076 & -.001332 & -.00006 \\
\hline Educación (12) & .0030841 & .0063727 & -.0028075 & \begin{tabular}{l|l}
5 & -.0004811
\end{tabular} & -.003891 & .0023854 & -.005926 & -.000351 \\
\hline Experiencia (13) & .0262077 & .0237297 & .0055951 & -.0031171 & .0280966 & .0305841 & .0028712 & -.005358 \\
\hline Experiencia $^{2}(14)$ & -.021692 & -.0195219 & -.0049672 & 2.0027964 & -.022606 & -.024363 & -.002004 & .0037607 \\
\hline Casado (15) & .000184 & -.0010328 & .0014307 & -.0002139 & -.009349 & -.000587 & -.009553 & .0007918 \\
\hline Jefe de hogar (16) & -.005142 & .0012224 & -.0071439 & .0007787 & -.010432 & .0012005 & -.012554 & .0009214 \\
\hline Tiempo completo (17) & -.001385 & .0006236 & -.0020407 & 7.0000321 & -.003006 & -.000241 & -.002749 & -.000016 \\
\hline
\end{tabular}

Fuente: Elaboración propia en base a los datos de la ENOE

tercer trimestre 2005, 2009 y 2015.

por género para el sector privado formal, cuadro 5, se aprecia una brecha salarial a favor de los hombres, misma que se reduce levemente a lo largo de todo el periodo, sin cambios relevantes entre subperidos y esta reducción se explica fundamentalmente por el comportamiento de los residuos salariales, renglón 3. La descomposición de la variación explicada en el tiempo permite identificar que en promedio las características favorecieron la reducción de la brecha salarial (renglón 7), mientras que el comportamiento promedio del precio de las mismas impulsa el aumento 


\section{Cuadro 4. Descomposición Juhn-Murphy-Pierce del diferencial salarial por razón de} género. Para el sector público. Tercer trimestre 2005-2009-2015.

(Los salarios por hora mensual están expresados a logaritmos)

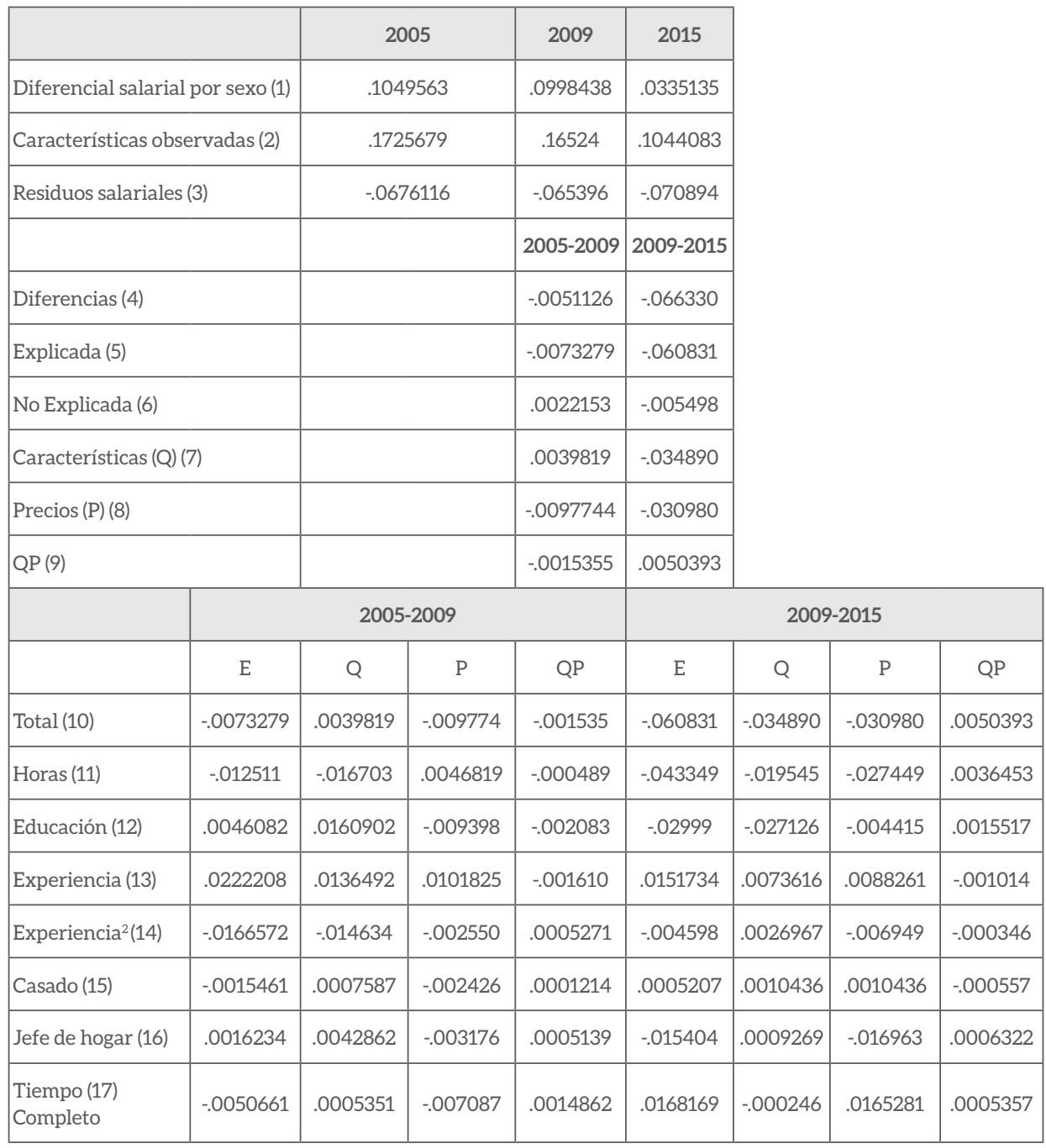

Fuente: Elaboración propia en base a los datos de la ENOE tercer trimestre 2005, 2009 y 2015.

de la brecha salarial (renglón 8). La parte inferior del cuadro 5 presenta la contribución al cambio de cada uno de los factores explicativos considerados en la estimación de la brecha salarial (renglones 10-17), identificando el aporte que se hace tanto por el cambio en la característica (Q) como por el precio (P), y en el caso del sector privado formal, se aprecian efectos encontrados en los factores educativos y experiencia, donde el primero contribuye a reducir la brecha salarial y el segundo a aumentarla; este comportamiento se aprecia en todo el periodo, y muestra que no obstante que la contribución neta de la parte explicada a la variación de la brecha 
salarial en el tiempo es poco relevante; al interior de factores explicativos se presentan modificaciones importantes.

Por último, en lo que se refiere al diferencial salarial por razón de género en el mercado de trabajo informal (cuadro 6), se observa que en este mercado al igual que en los anteriores se han reducido las brechas salariales. Cabe destacar, sin embargo, que en este caso, la caída es mayor que en los otros segmentos del mercado laboral. En el primer subperiodo, la reducción es de 0.06866 puntos logarítmicos, mientras en el último lustro el descenso es algo menor, pero de una magnitud similar (0.04341). Dada la importante presencia de mujeres en el mercado laboral privado informal, el comportamiento de la brecha salarial por género en la última década debe ser

\section{Cuadro 5. Descomposición Juhn-Murphy-Pierce del diferencial salarial por razón de género. Para el sector privado formal. Tercer trimestre 2005-2009-2015. \\ (Los salarios por hora mensual están expresados a logaritmos)}

\begin{tabular}{|c|c|c|c|c|c|c|c|c|}
\hline \multirow{2}{*}{\multicolumn{2}{|c|}{\begin{tabular}{|l} 
\\
Diferencial salarial por sexo (1)
\end{tabular}}} & 2005 & 2009 & 2015 & & & & \\
\hline & & -0.114218 & -0.10075 & $07-0.09297$ & & & & \\
\hline \multicolumn{2}{|c|}{ Características observadas (2) } & 0.0614434 & 0.0552592 & 0.055042 & & & & \\
\hline \multicolumn{2}{|c|}{ Residuos salariales (3) } & -0.175661 & -0.1560099 & -0.14802 & & & & \\
\hline & & & 2005-2009 & 2009-2015 & & & & \\
\hline \multicolumn{2}{|l|}{ Diferencias (4) } & & 0.0134678 & 0.0077724 & & & & \\
\hline \multicolumn{2}{|l|}{ Explicada (5) } & & -0.006184 & -0.000217 & & & & \\
\hline \multicolumn{2}{|l|}{ No Explicada (6) } & & 0.019652 & 0.0079896 & & & & \\
\hline \multicolumn{2}{|c|}{ Características (Q) (7) } & & 0.0125786 & 0.0001898 & & & & \\
\hline \multicolumn{2}{|l|}{ Precios (P) (8) } & & -0.018128 & -0.000109 & & & & \\
\hline \multicolumn{2}{|l|}{ QP (9) } & & -0.000634 & -0.000297 & & & & \\
\hline & \multicolumn{4}{|c|}{ 2005-2009 } & \multicolumn{4}{|c|}{ 2009-2015 } \\
\hline & $E$ & Q & $\mathrm{P}$ & QP & E & Q & $\mathrm{P}$ & QP \\
\hline Total (10) & -0.006184 & 0.0125786 & -0.0181283 & -0.000634 & -0.000217 & 0.0001898 & -0.000109 & -0.0002974 \\
\hline Horas (11) & -0.0023593 & -0.003824 & 0.0015405 & -0.0000759 & -0.0007011 & -0.0045592 & 0.0041069 & -0.0002487 \\
\hline Educación (12) & 0.0117681 & 0.0187985 & -0.0045564 & -0.002474 & -0.0094059 & -0.0056527 & -0.0042739 & 0.0005208 \\
\hline Experiencia (13) & 0.0276301 & 0.0136331 & 0.0166766 & -0.0026797 & 0.020581 & 0.02092 & -0.0005344 & 0.0001954 \\
\hline Experiencia $^{2}(14)$ & -0.0265233 & -0.018583 & -0.0109499 & 0.0030091 & -0.0140606 & -0.0157512 & 0.0027412 & -0.0010506 \\
\hline Casado (15) & -0.0094394 & 0.0005115 & -0.0109542 & 0.0010033 & -0.0004553 & 0.0016963 & -0.0024254 & 0.0002738 \\
\hline Jefe de hogar (16) & -0.0101464 & 0.0017408 & -0.0127718 & 0.0008846 & 0.0042414 & 0.0035379 & 0.0007818 & -0.0000784 \\
\hline $\begin{array}{l}\text { Tiempo } \\
\text { Completo (17) }\end{array}$ & 0.002886 & 0.0003011 & 0.0028869 & -0.0003019 & -0.0004167 & -0.0000013 & -0.0005057 & 0.0000903 \\
\hline
\end{tabular}

Fuente: Elaboración propia en base a los datos de la ENOE tercer trimestre 2005, 2009 y 2015. 
considerado como un signo de mayor equidad laboral en el mercado laboral mexicano, especialmente porque la fuente de la reducción proviene de la parte asociada a factores inobservables (renglón 6) dentro de los cuáles se encuentra la discriminación.

En resumen, el diferencial salarial por razón de género se ha reducido en México en todos los segmentos del mercado de trabajo considerados durante el período analiza- do. Existen, sin embargo, diferencias entre ellos en lo que se refiere a la magnitud de la brecha y a la evolución entre los distintos subperiodos de estudio. En concreto, se observan disminuciones más fuertes en el primer lustro respecto al segundo, mientras que por tipo de mercados, el privado informal es el que presenta mayor reducción, aunque también es el que tiene mayor diferencia salarial no asociada a las características. Se constata que las diferencias en las

\section{Cuadro 6. Descomposición Juhn-Murphy-Pierce del diferencial salarial por razón de género. Para el sector privado informal. Tercer trimestre 2005-2009-2015.}

(Los salarios por hora mensual están expresados a logaritmos)

\begin{tabular}{|c|c|c|c|c|c|c|c|c|}
\hline & & 2005 & 2009 & \multicolumn{2}{|l|}{2015} & & & \\
\hline \multicolumn{2}{|c|}{ Diferencial salarial por sexo (1) } & -.163169 & -.0945036 & \multicolumn{2}{|c|}{-.051093} & & & \\
\hline \multicolumn{2}{|c|}{ Características observadas (2) } & .1299011 & .1392027 & \multicolumn{2}{|c|}{.1481907} & & & \\
\hline \multicolumn{2}{|l|}{ Residuos salariales (3) } & -293070 & -.2337063 & \multicolumn{2}{|c|}{-199284} & & & \\
\hline & & & 2005-2009 & \multicolumn{2}{|c|}{ 2009-2015 } & & & \\
\hline \multicolumn{2}{|l|}{ Diferencias (4) } & & .0686658 & \multicolumn{2}{|c|}{.0434099} & & & \\
\hline \multicolumn{2}{|l|}{ Explicada (5) } & & .0093016 & \multicolumn{2}{|c|}{.008988} & & & \\
\hline \multicolumn{2}{|l|}{ No Explicada (6) } & & .0593643 & \multicolumn{2}{|c|}{.0344219} & & & \\
\hline \multicolumn{2}{|l|}{ Características (Q) (7) } & & .0064689 & \multicolumn{2}{|c|}{.0308183} & & & \\
\hline \multicolumn{2}{|l|}{ Precios (P) (8) } & & .0040578 & \multicolumn{2}{|c|}{-.020691} & & & \\
\hline \multicolumn{2}{|l|}{$\mathrm{QP}(9)$} & & -.001225 & \multicolumn{2}{|c|}{-.001138} & & & \\
\hline & \multicolumn{4}{|c|}{ 2005-2009 } & \multicolumn{4}{|c|}{ 2009-2015 } \\
\hline & E & Q & $\mathbf{P}$ & QP & $\mathrm{E}$ & Q & $\mathbf{P}$ & QP \\
\hline Total (10) & .0093016 & .0064689 & .0040578 & -.001225 & .008988 & .0308183 & -.020691 & -.001138 \\
\hline Horas (11) & .0057093 & .0017028 & .0039707 & .0000359 & .0277411 & .0161425 & .0107084 & .0008902 \\
\hline Educación (12) & -.001206 & -.001364 & $-6.58 e-06$ & .0001639 & .0096164 & .0116162 & .0002202 & -.002219 \\
\hline Experiencia (13) & .0158388 & .0192162 & -.00015 & -.003227 & .0157407 & .0248077 & -.003652 & -.005414 \\
\hline Experiencia ${ }^{2}(14)$ & -.009866 & -.012208 & .0000851 & .0022568 & -.013609 & -.020736 & .0023694 & .004758 \\
\hline Casado (15) & .0026243 & -.001385 & .0050789 & -.001068 & -.008456 & -.000699 & -.008394 & .0006373 \\
\hline Jefe de hogar(16) & -.003628 & .0012005 & -.0054369 & .000608 & -.006802 & .000895 & -.008209 & .0005115 \\
\hline Tiempo (17) Completo & -.000169 & -.000692 & .0005165 & $6.52 \mathrm{e}-06$ & -.015242 & -.001207 & -.013733 & -.000301 \\
\hline
\end{tabular}


dotaciones de características productivas entre mujeres y hombres, capturadas por el primer término de la descomposición, justifica una proporción relativamente menor de la desigualdad excepto para el sector público. Los factores inobservables tienen una capacidad explicativa del diferencial salarial promedio por género significativa y mayor que la parte explicada. Sin embargo, se observa una reducción en el tiempo principalmente de 2005-2009.

\section{CONCLUSIONES}

En este trabajo se han analizado las diferencias salariales por razón de género en México y en diferentes segmentos de su mercado laboral: público y privado (formal e informal) durante la última década. El análisis se desarrolla a través de la metodología de descomposición de Oaxaca-Blinder (1973) y de Juhn-MurphyPierce (1991, 1993).

Los resultados indican que existe una brecha salarial por género, y que su magnitud y dirección es heterogénea: en el sector público es favorablea las mujeres y en el privado las desfavorece, particularmente en el sector informal. La descomposición de la brecha salarial permite identificar en el caso del sector público que la fuente principal de brecha salarial por género proviene de la parte explicada, mientras en el sector privado, las diferencias se asocian a la parte no explicada. Este último resultado parece indicar una mayor presencia de discriminación salarial en los segmentos privados formal e informal del mercado laboral.

Durante la última década la brecha salarial se redujo, mostrando una mayor intensidad en el primer lustro y aunque no presenta diferencias relevantes entre los subperiodos a pesar de la presencia de perturbaciones económicas relevantes a mitad del periodo analizado, lo que nos permite afirmar que a partir de los resultados obtenidos, noes posible sostener la presencia de una brecha salarial con comportamiento cíclico, como lo sostienen los autores señalados en la revisión teórica, y en su lugar encuentra mayor respaldo la hipótesis de Becker, vinculado al hecho de que la competencia reduce la discrepancia en las remuneraciones salariales, no solo porque se aprecia una reducción en la brecha salarial por sexo, sino porque la fuente de la menor diferencia se asocia con una caída en la parte no explicada y que generalmente puede asociarse a la presencia de discriminación salarial.

Otros hechos a destacar son: i) en el sector público existe un esquema de remuneración relativa más acorde a los principios de dotaciones de capital humano; ii) la mayor reducción de la brecha salarial y de las diferencias no explicadas se encuentran en el sector informal, donde los salarios son más bajos y existe una mayor presencia de mujeres; muchas de ellas jefas de hogar y aportantes únicas o principales de recursos económicos del hogar.

No obstante, y pese a su reducción, no se puede soslayar que la principal fuente de inequidad por género corresponde a la parte no explicada, y, por tanto se deben continuar implementando políticas públicas tendientes a generar una mayor equidad remunerativa por sexo. Por último, el estudio presenta dos limitaciones importantes que se abordarán en investigaciones futuras: en primer lugar, no se analizan (debido a las limitaciones de la base de datos) las decisiones de participación en el mercado de trabajo y de selección en el sector público y privado formal e informal. En segundo lugar, también sería interesante analizar la evolución de las diferencias salariales en- 
tre hombres y mujeres a lo largo de toda la distribución y no solo en la media como se ha realizado aquí con el objetivo de valorar si los efectos de ciclo son heterogéneos a lo largo de dicha distribución (Popli, 2013; Arceo y Campos, 2014).

\section{REFERENCIAS BIBLIOGRÁFICAS}

Alarcón D. y McKinley T. (1994). "Gender differences in wages and human capital: case study of female and male urban workers in México from 1984 to 1992". Frontera Norte, 6(12): 41-50.

Arceo E. y Campos R. (2014). "Evolución de la brecha salarial de género en México". El Trimestre Económico, Núm. 323, pp. 619-653.

Artecona R. y Cunningham W. (2002). "Effects of trade liberalization on the gender wage gap in México". Gender and Development Working Paper Series 21. The World Bank.

Becker G. (1971), The Economic of Discrimination. Second Edition. The University of Chicago Press. Pp.167.

Bertrand, M. (2011). "New perspectives on gender", en David Card y Orley Ashenfelter (eds.), Handbook of Labor Economics, Elsevier, pp. 1543-1590.

Biddle, J. y Hamermesh, D. (2013), "Wage discrimination over the business cycle", IZA Journal of Labor Policy, 2:7.

Blinder A. (1973). "Wage discrimination: reduced forms and structural estimates". Journal of Human Resourses, 8(4). The University of Texas at Austin. Pp. 436-455.

Brown F. y Domínguez L. (2007). "Determinants of wage differentials in the maquila industry in México: a gender perspective". Working Paper, Núm. 07-6, The International working group on GenderMacroeconomic-International Economic. Salt Lake City. Pp.1-24.

Brown, C., Pagan J. y Rodríguez-Oreggia E. (1999). “Ocupational attainment and gender earning differentials in Mexico". Industrial and Labour Relations Review, 53(1): 123-135.

Calónico S. y Ñopo H. (2008). "Gender segregation in the workplace and wage gaps: Evidence from urban México 1994-2004". In J. Bishop (ed). Ocupational and Residential segregation (Research on Economic Inequality). Vol. 17. Emerald Group Publishing limited.

Castro D., Huesca L. y Zamarrón N. (2015). "Discriminación salarial por género, en la industria manufacturera de la frontera norte de México, en el periodo 2005-2011", Nóesis. Revista de Ciencias Sociales y Humanidades, vol. 24, núm. 47, enero-junio, pp. 50-80 Instituto de Ciencias Sociales y Administración Ciudad Juárez, México

Cuevas A, M. Messmacher y A.M. Werner (2003). "Sincronización macroeconómica entre México y sus socios comerciales del TLCAN”. Documento de investigación No. 2003-01. Banco de México.

Delajara Marcelo (2012). "Sincronización entre los ciclos económicos de México y Estados Unidos. Nuevos resultados con base en el análisis de los índices coincidentes regionales de México". Documento de investigación No. 2012-01. Banco de México.

De la Roca J. (2014) "Wage cyclicality: Evidence from Spain using social security data" Working Paper New York University, pp. 1-22.

Del Razo L. (2003). "Estudio de la brecha salarial entre hombres y mujeres en México (1994-2001)". Documentos de Investigación, Núm. 4, Secretaría de Desarrollo Social, pp. 41. 
DiNardo J.; Fortin N. y Lemieux T. (1996). "Labor market institutions and the distribution of wages, 1973-1992: A Semiparametric Approach”. Econometrica. 64(5): 1001-1044.

Eswaran, M. (2014). Why gender matters in economics, Princeton University Press.

Félix Gustavo y Dávila Alejandro. (2008). "Apertura comercial y demanda en el crecimiento de las entidades federativas en México". Comercio Exterior. Vol. 58. Num.4. 258-270.

Finio, Nicholas J. (2010) "The T rend of the Gender Wage Gap Over the Business Cycle," Gettysburg Economic Review: Vol. 4, Article 5.

Ghosh J. (2013). Economic crises and women's work: exploring progressive strategies in a rapidly changing global environment, UN Women, pp. 58.

Instituto Nacional de Estadística y Geografía (2012). Mujeres y hombres en México 2012. XXIV, PP.169.

Instituto Nacional de Estadística y Geografía. 2005-2014. Encuesta Nacional de Ocupación y Empleo. INEGI, Aguascalientes, México.

Instituto Nacional de Estadística y Geografía (2010). Censo de Población. INEGI, Aguascalientes, México.

Juhn, C., Murphy K. y Pierce B. (1991). "Accounting for the Slowdown in Black-White Convergence", en M. Osters (ed.) Workers and Their Wages, ed. American Enterprise Institute Press.

Juhn, C., Murphy K. y Pierce B. (1993). "Wage inequality and the rise in returns to skill”. Journal of Political Economy, 101 (31), pp. 410-442.

Kandill M. y Woods J. (2002) "Convergence of the gender gap over the Business cycle: a sectorial investigation", Journal of Economics and Business, 54, 271-292.

King A. y Owen J. (2009). "Asia in the Global Economic Crisis: Impacts and response from a gender perspective", Technical Note, ILO, Asia Decent Work Decade 2006-2015, pp. 23.

McConell C., Brue S. y Macpherson D. (2003), Economía laboral, Sexta Edición, McGraw-Hill/ Interamericana de España, Barcelona.

Oaxaca R. (1973). "Male-female wage differentials in urban labour markets". International Economic Review, 14 (3), University of Pennsylvania-The Osaka University Philadelphia. Pp. 693-709.

Oaxaca R. y Ramson M. (1994), "On discrimination and the decomposition of wage differentials", Journal of Econometric, Vol. 61, Pp. 279-295.

OCDE (2013). Better life index. http://www.oecdbetterlifeindex.org/es/countries/united-states-es/.

O'Neill, J. (1985). The trend in the male-female wage gap in the United States. Journal of Labor Economics, 3, S91-S116.

Park S. y Shin D. (2005). "Explaining procyclical male-female wage gap", Economics Letters, 88, pp. 231-235.

Peña-Boquete Y. (2014), "Have the economic crises reduced the gender gap on the Spanish labour market?”, Revue de l'OFCE 2014/2 (Num. 133), p. 277-302.

Popli G. (2013). "Gender wage differentials in México: A distributional approach". Journal of the Royal Statistical Society, A. Vol. 176 (2), Statics in Society, London, Pp. 295-319. 
Robinson J. (1933). The Economic of Imperfect Competition. New York: St Martin's Press.

Razzu G. y Singleton C. (2016). "Gender and the business cycle: An analysis of labour markets in the US and UK, Journal of Macroeconomics, 1-16.

Rodríguez R. y Castro D. (2014a). "Discriminación salarial de la mujer en el mercado laboral de México y sus regiones”, Revista Economía, Sociedad y Territorio. Vol. XIV. Núm. 46, Pp. 655-686.

Rodríguez R. y Castro D. (2014b). "Análisis de la discriminación salarial por género en Saltillo y Hermosillo: Un estudio comparativo en la industria manufacturera". Nóesis, Revista de ciencias sociales y humanidades, UACJ, Vol. 23. Núm. 46. Pp. 80-113.

Simón H., Ramos R. y Sanromá E. (2008). "Evolución de las diferencias salariales por razón de sexo", Revista Economía Aplicada, Vol. 16, Núm. 48, Pp. 37-68.

Tenjo, J.; Rivero, R. y Bernat, L. (2005). "Evolución de las diferencias salariales por sexo en seis países de América Latina un intento de interpretación”. Documento Cede 2005-18, Universidad de los Andes, Bogotá, D. C.

World Economic Forum (2015), The Global Gender Gap Report 2015, http://reports.weforum.org/ global-gender-gap-report-2015/economies/\#economy=MEX

Yun, M. (2005), "A Simple Solution to the Identification Problem in Detailed Wage Decompositions", Economic Inquiry, Vol. 43, pp. 766-772.

\section{Para citaciones:}

Rodríguez-Pérez, R., Ramos-Lobo, R., \& Castro-Lugo, D. (2017). Brecha salarial por género en los mercados de trabajo público y privado en México. Panorama Económico, 25, 2, pp. 149-172.

\section{AUTORES}

Reyna Rodríguez-Perez

Profesora de Tiempo completo de la Facultad de Economía de la Universidad Autónoma de Coahuila (UAdeC), Unidad Campo redondo edificio "E" planta baja, CP 25000. Saltillo, Coahuila. Doctora en Ciencias por el Centro de Investigación en Alimentación y Desarrollo (CIAD)

Raúl Ramos-Lobo

Profesor Asociado e Investigador Senior del Departamento de Econometría, Estadística y Economia Aplicada de la Universidad de Barcelona (España). Ph.D. en Economía y Ciencias de la Gestión de la Universidad de Barcelona (España)

\section{David Castro-Lugo}

Profesor e Investigador del Centro de Investigaciones Socioeconómicas de la Universidad Autónoma de Coahuila (México), Unidad Camporredondo Edificio "S", CP 25080, Saltillo, Coahuila. Ph.D. en Economía de la Universidad Autonóma de Barcelona (España) 